\title{
The effect of supervised dynamic exercise program on somatosensory temporal discrimination in patients with fibromyalgia syndrome
}

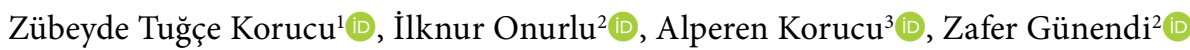 \\ ${ }^{1}$ Department of Physical Medicine and Rehabilitation, Ankara City Hospital, Ankara, Turkey \\ ${ }^{2}$ Department of Physical Medicine and Rehabilitation, Gazi University Faculty of Medicine, Ankara, Turkey \\ ${ }^{3}$ Department of Orthopedics and Traumatology, Ankara City Hospital, Ankara, Turkey
}

\section{ABSTRACT}

Objectives: This study aims to investigate the effect of a supervised dynamic exercise program on somatosensory temporal discrimination (STD) which indicates the central sensory processing ability.

Patients and methods: Forty-eight patients (mean age: 43 years; range, 19 to 64 years) with fibromyalgia syndrome (FMS) were included in this prospective, randomized study between April 2018 and October 2018. Demographics and clinical measurements were recorded. The STD threshold was measured by a researcher blinded to the treatment groups. After baseline assessments, the patients were randomized into two groups: the supervised exercise group (SEG) and the home exercise group (HEG) as the control group. The SEG performed submaximal aerobic exercise and low-medium resistant isotonic exercises under the supervision of a physiotherapist, 1-h per day, three days in a week for four weeks. The HEG was given a home-based exercise program consisting of low-to-medium resistance isotonic exercises and aerobic exercises 1-h per day, three days in a week for four weeks. After exercise program, clinical assessments and STD threshold measurement were repeated.

Results: Clinical and STD threshold measures improved significantly after four-week exercise program in both groups. The improvements in clinical measures were significantly higher in the SEG. However, there was no difference in the STD threshold improvement between the groups. No correlation was found between the STD threshold measure and clinical features in any patients.

Conclusion: Although a significant improvement was achieved in impaired STD threshold with SEG, it was not found to be superior to the amelioration observed with HEG.

Keywords: Exercise, fibromyalgia, somatosensory temporal discrimination.

Fibromyalgia syndrome (FMS) is a disease characterized by specific clinical findings such as chronic pain, sleep disorders, and cognitive disorders, the cause of which is not fully known, but which is seen more widely in females. ${ }^{1}$ Considering the etiopathogenesis and symptoms, it is thought to be a central sensitization syndrome. ${ }^{1}$

A multidisciplinary approach is the basis of treatment for FMS. In practice, pharmacological treatments are applied together with nonpharmacological treatment approaches such as physical therapy, exercise, and cognitivebehavioral therapy. Aerobic, strengthening, stretching and relaxation exercises and aquatic exercises have been shown to be of benefit in FMS. Exercise is recommended as one of the treatment options that has always been most accepted. The positive effect of exercise for FMS

Received: August 26, 2020 Accepted: December 22, 2020 Published online: February 082021

Correspondence: Zafer Günendi. MD. Ankara Şehir Hastanesi Ortopedi ve Travmatoloji Kliniği, 06800 Çankaya, Ankara, Türkiye. Tel: +90 312 - 2025219 e-mail: zafergunendi@yahoo.com 
patients is thought to be through the central nervous system rather (CNS) than as a peripheral effect. ${ }^{2,3}$

Somatosensory temporal discrimination (STD) is the perception of two separate stimuli applied to the body with a short time interval. This is a purely sensory process that is useful in the processing of information coming from external sources. The STD function requires a high cognitive process. The functionality of CNS areas such as basal ganglions and the primary somatosensorial cortex for STD is needed. ${ }^{4,5}$ The STD threshold (STDT) value measurement is used to measure the STD ability of individuals. These values have been found to be significantly higher in fibromyalgia, which is a central sensitization syndrome. ${ }^{6}$ Therefore, the STD measurement can be used as a neurophysiological marker of the efficacy of fibromyalgia treatment.

In the present study, we aimed to investigate the effect of a supervised dynamic exercise program on STD, which indicates the central sensory processing ability.

\section{PATIENTS AND METHODS}

This single-blind, prospective, randomizedcontrolled study included a total of 48 female FMS patients (mean age: 43 years; range, 19 to 64 years) diagnosed according to the 2010 American College of Rheumatology (ACR) classification criteria who presented at the outpatient clinic of the Physical Medicine and Rehabilitation Department of Gazi University Faculty of Medicine between April 2018 and October 2018. Inclusion criteria were as follows: age between 18 and 65 years, with at least eight years of formal education, and chronic pain of an intensity of $\geq 4 \mathrm{~cm}$ on a $0-10 \mathrm{~cm}$ Visual Analog Scale (VAS) for pain within the past seven days. Exclusion criteria included secondary fibromyalgia, intellectual deficits, central and peripheral neurological disorders that may affect sensory processing such as stroke, epilepsy, headache disorders, peripheral neuropathy, the use of any medication affecting the CNS and inflammatory rheumatic diseases and orthopedic problems that can affect exercise participation. A written informed consent was obtained from each patient. The study protocol was approved by the Gazi University Faculty of Medicine Ethics
Committee (Approval Date/No: 04.2018/270). The study was conducted in accordance with the principles of the Declaration of Helsinki.

\section{Clinical assessment}

Age, sex, height, weight, body mass index, time since diagnosis of the disease, marital status, level of education, occupational characteristics, medications used and any comorbid diseases of each participants were recorded. All patients were evaluated at baseline and after four weeks of treatment. The VAS for pain, Hospital Anxiety and Depression Scale (HADS) (Anxiety: HAD-A, Depression: HAD-D), Fibromyalgia Impact Questionnaire (FIQ), and Symptom Severity Scale (SSS) were used for clinical assessment. Additionally, the STDT was measured by a researcher blinded to the treatment groups.

The patient's psychological state was assessed by using the HADS. This scale consists of 14 items, seven measuring anxiety (HAD-A) and seven measuring depression (HAD-D). Each question is scored on a four-point (0-3) scale, where a higher score represents more severe depression or anxiety. The Turkish version of HADS was found to be valid and reliable. ${ }^{?}$

The FIQ was used to assess function and health-related quality of life of patients. This self-administered questionnaire evaluates pain, tenderness, fatigue, stiffness, multidimensional function, and sleep. Final score ranges between 0 and 80. High scores indicate major impact of fibromyalgia on functioning. The Turkish version of the FIQ was shown to be valid and reliable. ${ }^{8}$

The SSS was used to evaluate symptom severity for fatigue, sleep quality, cognitive symptoms, and somatic symptoms. The severity of these symptoms over the past week were rated on a scale from 0 to 3. The final SSS is the sum of the severity of these symptoms and ranges between 0 and $12 .{ }^{9}$ The Turkish version of SSS for fibromyalgia was found to be valid and reliable. ${ }^{10}$

\section{STD assessment}

The procedures defined in previous studies were applied to measure STDT values. ${ }^{4,6}$ The measurements were taken from the dorsum of the dominant hand of the fibromyalgia patients. First, the skin was cleaned with alcohol, then anodal and cathodal $\mathrm{Ag}-\mathrm{AgCl}$ skin electrodes of 
$10 \mathrm{~mm}$ in diameter were placed at a distance of approximately 2-cm apart. A fixed current stimulus was used (Keypoint ${ }^{\circledR}$; Medtronic Functional Diagnostics, Denmark). The duration of each electric current was $0.2 \mathrm{msn}$. The maximum current intensity was previously set at $10 \mathrm{~mA}$. To determine the minimal stimulus intensity, a stimulus of $1 \mathrm{~mA}$ was the initial stimulus and this was gradually increased in stages of $0.2 \mathrm{~mA}$. The minimal sensory threshold value measurement was repeated three times. The stimulus intensity which is felt consistently in these three trials was recorded as the minimum sensory threshold. The first interstimulus interval (ISI) between matched stimuli was $5 \mathrm{msn}$ and this was increased in steps of $5 \mathrm{msn}$. The first of three consecutive ISIs, when the patient was able to differentiate between two separate stimuli was recorded as the ascendant STDT (aSTDT). In the continuation of the test, three more stimuli were given at longer gaps than the threshold ISI and the persistence of paired stimuli was accepted as a significant STD value. Then, the stimulus administered was reduced at $5 \mathrm{msn}$ intervals, until the patient felt it as a single stimulus and this value was recorded as the descendent STDT ( $\mathrm{dSTDT})$. The measurements were repeated at 1.5-fold minimal sensory threshold severity. The arithmetic mean of two values was calculated and recorded as the STDT value for the dominant hand. To prevent habituation, the stimuli were given at random intervals.

\section{Exercise procedure}

The patients were randomized into two equal groups of 24 as the supervised exercise group (SEG) and the home exercise group (HEG). Simple randomization was performed using a computer-generated random number list. Those included in SEG were given an exercise program that consisted of submaximal aerobic exercise (treadmill) and low-medium resistant isotonic exercises (posture exercises, and shoulder strengthening exercises with neck joint range of movement and a slightly resistance band) under the supervision of a physiotherapist, 1-h per day, three days in a week for four weeks. Those included in HEG were given a home exercise (HE) program that consisted of low-to-medium resistance isotonic exercises and aerobic exercises (for the exercises to be the same as for the SEG) 1-h per day, three days in a week for four-weeks. A physiotherapist explained how the exercise program was to be applied with the support of a detailed written and illustrated exercise form. The patients were also recommended to undertake aerobic exercise in the form of walking three days a week. After exercise program, clinical assessments and STDT measurement were repeated.

It was planned to include 20 FMS patients in each group to achieve a mean difference of $25 \pm 27 \mathrm{msn}$ in STDT change between groups with an alpha error of 0.05 and $80 \%$ power. ${ }^{4}$ Due to the possibility of a $20 \%$ dropout rate, 24 patients were included in each group. The flowchart of patient inclusion is shown in Figure 1.

\section{Statistical analysis}

Statistical analysis was performed using the IBM SPSS version 22 (IBM Corp., Armonk, NY, USA). Descriptive data were expressed in mean \pm

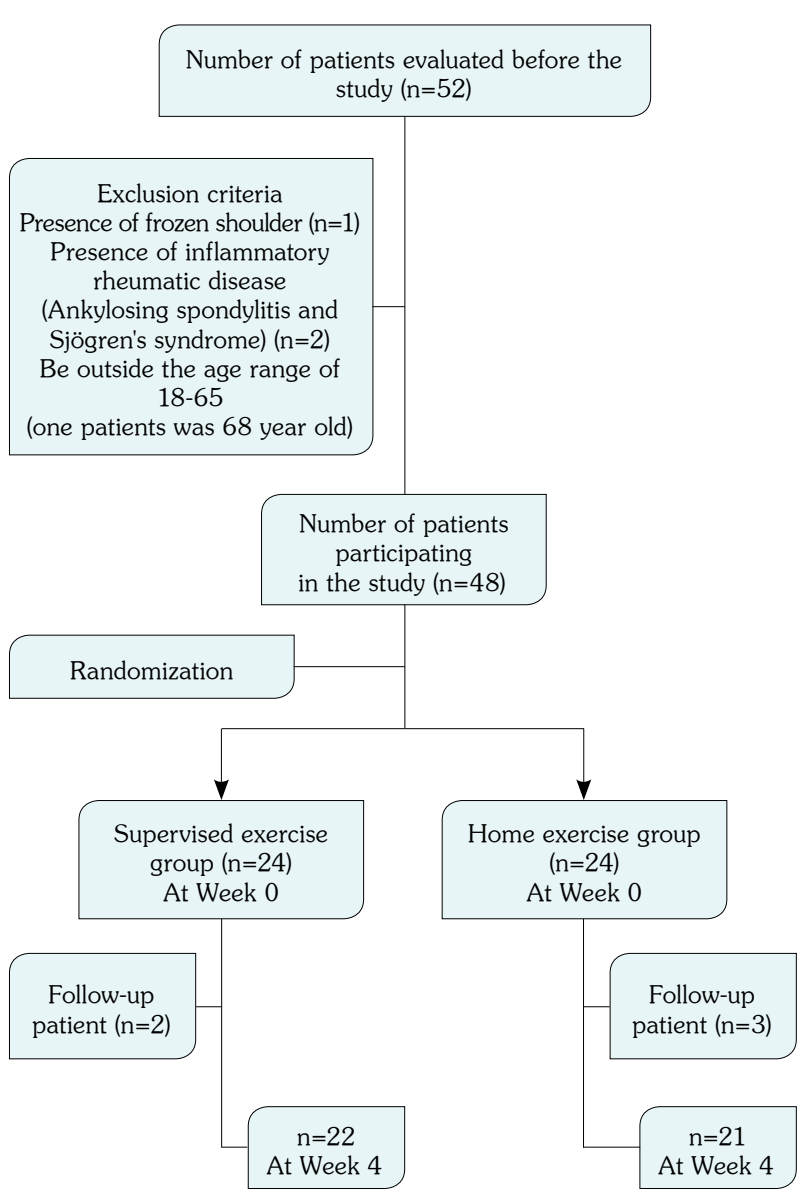

Figure 1. Study flow chart. 
standard deviation (SD), median and interquartile range (IQR) with 95\% confidence interval (CI). Conformity of the data to normal distribution was assessed using the Kolmogorov-Smirnov test. In the comparisons of demographic, baseline clinical and neurophysiological variables, the independent samples t-test was used for data with normal distribution and the Mann-Whitney $U$ test was used for data not showing normal distribution. In the evaluations of the relationships of the baseline demographic, clinical, and neurological data of all the patients, the Pearson or Spearman correlation coefficient was used according to the normality of the data. To evaluate the effect of the treatment on the final results, two-way analysis of variance in repeated measurements was applied (treatment group $\times$ time). The data of patients who withdrew from treatment during follow-up was evaluated with the intention-to-treat analysis using the last observation carried forward (LOCF) approach. A $p$ value of $<0.05$ was considered statistically significant.

\section{RESULTS}

No statistically significant difference was found between the groups in respect of demographic data, baseline pain severity, HAD-A, HAD-D, FIQ, SSS scores, and STDT (Table 1).

The changes over time from baseline to the end of the fourth week in the pain severity, HAD-A, HAD-D, FIQ, SSS and STDT values of both groups are shown in Table 2. A statistically significant change over time was observed in all the parameters evaluated in both groups (Table 2).

In the two-way variance analysis of the repeated measurements, a statistically significant difference was found in the treatment group $\times$ time interaction in favor of SEG in VAS, HAD-A, HAD-D, FIQ, and SSS (F[1.46]:6.471, 7.79, 7.028, 10.509, 5.686; $\mathrm{p}=0.014, \mathrm{p}=0.008, \mathrm{p}=0.011, \mathrm{p}=0.002$, and $\mathrm{p}=0.021$, respectively) (Figures $2 \mathrm{a}-\mathrm{c}, 3 \mathrm{a}, \mathrm{b}$ ). No statistically significant difference was found in the treatment group $\times$ time interaction in STDT ( $F[1.46]: 1.884 ; p=0.177$ ) (Figure 3c).

The correlations between the demographic characteristics, baseline clinical and neurophysiological evaluations of all the patients are shown in Table 3. A significant negative correlation was found between age and the HAD-A

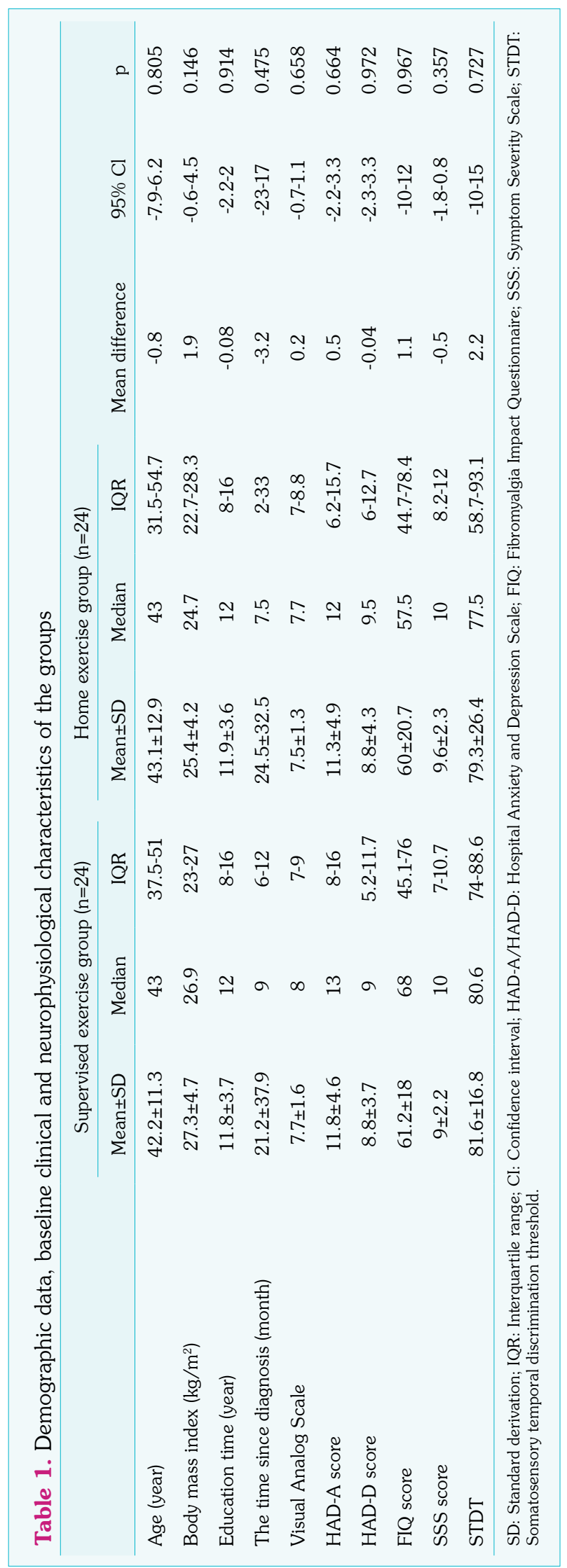




\begin{tabular}{|c|c|c|c|c|c|c|}
\hline & \multicolumn{3}{|c|}{ Supervised exercise group $(n=24)$} & \multicolumn{3}{|c|}{ Home exercise group $(n=24)$} \\
\hline & Mean \pm SD & Median & IQR & Mean \pm SD & Median & IQR \\
\hline \multicolumn{7}{|c|}{ Visual Analog Scale } \\
\hline Baseline & $7.7 \pm 1.6$ & 8 & $7-9$ & $7.5 \pm 1.3$ & 7.7 & $7-8.8$ \\
\hline At Week 4 & $3.6 \pm 1.9$ & 3 & $2-5$ & $4.7 \pm 2.1$ & 4 & $3-6.7$ \\
\hline$p$ & $<0.001$ & & & $<0.001$ & & \\
\hline \multicolumn{7}{|l|}{ HADA score } \\
\hline Baseline & $11.8 \pm 4.6$ & 13 & $8-16$ & $11.3 \pm 4.9$ & 12 & $6.2-15.7$ \\
\hline At Week 4 & $6.08 \pm 3.5$ & 5 & $3-8$ & $8.2 \pm 4.8$ & 6.5 & $4.2-11.7$ \\
\hline$p$ & $<0.001$ & & & $<0.001$ & & \\
\hline \multicolumn{7}{|l|}{ HADD score } \\
\hline Baseline & $8.8 \pm 3.7$ & 9 & $5.2-11.7$ & $8.8 \pm 4.3$ & 9.5 & $6-12.7$ \\
\hline At Week 4 & $4.5 \pm 1.9$ & 4 & $3.25-8$ & $6.6 \pm 4$ & 6 & 4-9.5 \\
\hline$p$ & $<0.001$ & & & $<0.001$ & & \\
\hline \multicolumn{7}{|l|}{ FIQ score } \\
\hline Baseline & $61.2 \pm 18$ & 68 & $45.1-76$ & $60 \pm 20.7$ & 57.5 & 44.7-78.4 \\
\hline At Week 4 & $30.5 \pm 16.7$ & 29 & $17.6-41.7$ & $43.2 \pm 18.5$ & 40.4 & $27.7-55.5$ \\
\hline$p$ & $<0.001$ & & & $<0.001$ & & \\
\hline \multicolumn{7}{|l|}{ SSS score } \\
\hline Baseline & $9 \pm 2.2$ & 10 & $7-10.7$ & $9.6 \pm 2.3$ & 10 & $8.2-12$ \\
\hline At Week 4 & $4.9 \pm 2.05$ & 4 & $4-6.7$ & $6.8 \pm 2.4$ & 7 & $4.2-8.7$ \\
\hline$p$ & $<0.001$ & & & $<0.001$ & & \\
\hline \multicolumn{7}{|l|}{ STDT } \\
\hline Baseline & $81.6 \pm 16.8$ & 80.6 & $74-88.6$ & $79.3 \pm 26.4$ & 77.5 & $58.7-93.1$ \\
\hline At Week 4 & $73.3 \pm 16$ & 73.1 & $66.8-80.9$ & $73.3 \pm 24.9$ & 72.5 & $53.7-89$ \\
\hline n & $<0.001$ & & & $<0.001$ & & \\
\hline
\end{tabular}

score and a positive correlation between age and the STDT value $(\mathrm{r}=-0.36, \mathrm{p}=0.01 ; \mathrm{r}=0.29, \mathrm{p}=0.04$ respectively). A significant negative correlation was shown between the level of education and the VAS, HAD-D, and FIQ scores $(\mathrm{r}=-0.44, \mathrm{p}=0.001$; $\mathrm{r}=-0.51, \mathrm{p}<0.001 ; \mathrm{r}=-0.31, \mathrm{p}=0.03$ respectively). There was a significant correlation between the time since diagnosis and the SSS score $(\mathrm{r}=0.31$, $p=0.03$ ). The clinical parameters (VAS, HADA, HAD-D, FIQ, and SSS) were found to be significantly correlated with each other. The STDT showed a significant correlation with age only $(r=0.29, p=0.04)$.

\section{DISCUSSION}

The results of this study demonstrated that a short-term supervised dynamic exercise program including aerobic and low-moderate resistance strengthening exercises had a positive effect on STD ability, in addition to its positive effect on clinical parameters such as pain, fatigue, mood status, functionality in patients with FMS. However, we observed no significant difference in the STDT between the SEG and HEG.

Several studies have shown the effect of aerobic and strengthening exercises on the clinical parameters of fibromyalgia, and exercise is the primary recommendation in international guidelines for FMS treatment. ${ }^{2,3}$ Exercise increases muscle strength and resistance, achieves appropriate posture, has positive effects on pain and mood, and improves function and quality of life. These effects occur through peripheral mechanisms and much more through central mechanisms. In the current study, both the four-week $\mathrm{HE}$ and supervised exercise (SE) program had positive effects on clinical symptoms and findings when evaluated with the VAS, HAD-A, HAD-D, FIQ, and SSS. In addition, when the treatment group $\times$ time interaction was 
(a)

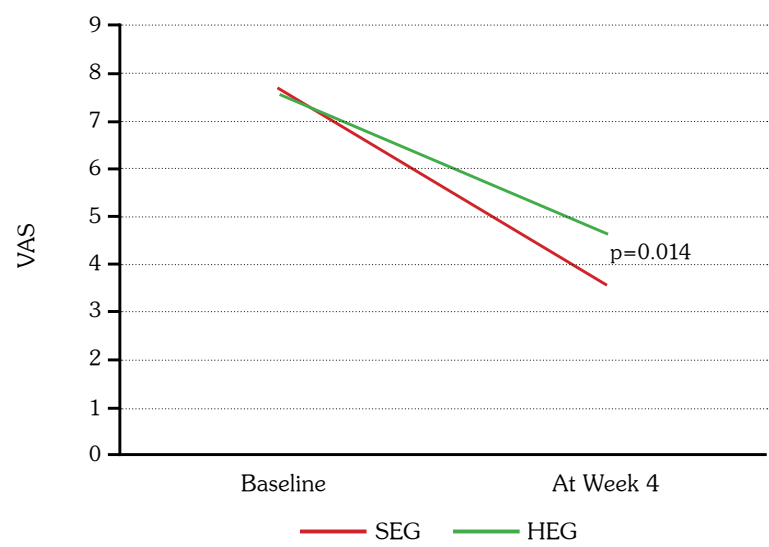

(b)



(c)



Figure 2. (a-c) Changes between the pain measurements at baseline and at Week 4. HAD-A. HAD-D scores in both groups.

SEG: Supervised exercise group; HEG: Home exercise group; VAS: Visual Analog Scale; HAD-A: Hospital Anxiety and Depression Scale-Anxiety; HAD-D: Hospital Anxiety and Depression Scale-Depression.

evaluated, the improvement in clinical parameters was determined to be significantly greater in the SEG. (a)

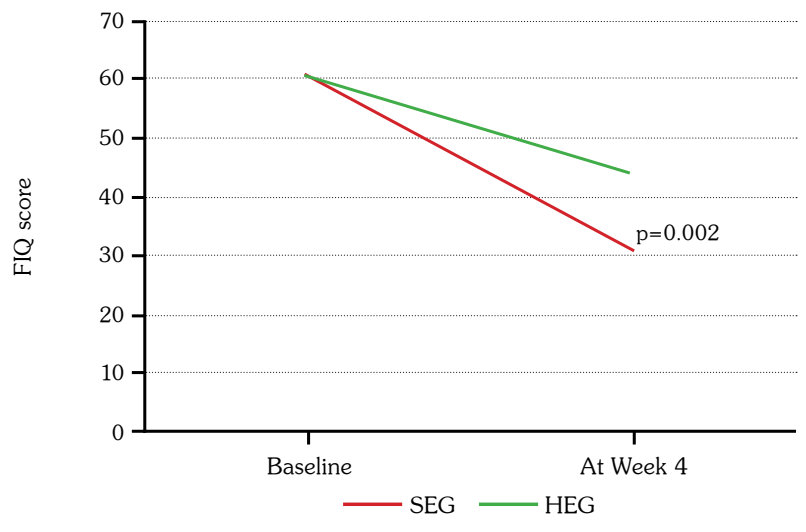

(b)

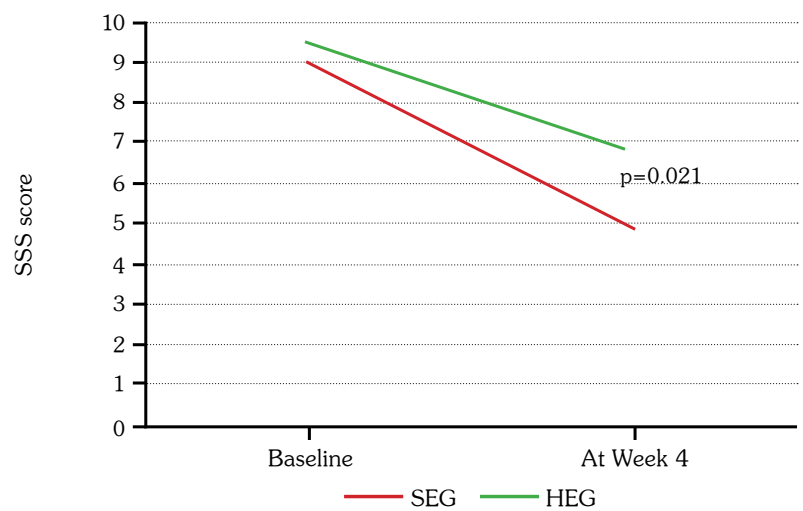

(c)

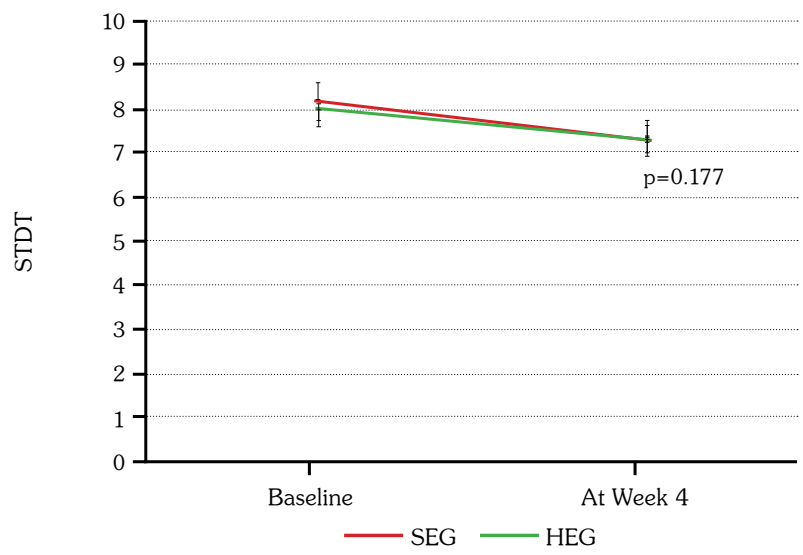

Figure 3. (a-c) Changes between baseline and $4^{\text {th }}$ Week measurements in FIQ and SSS scores and STDT measures in both groups.

SEG: Supervised exercise group; HEG: Home exercise group; FIQ: Fibromyalgia Impact Questionnaire; SSS: Symptom Severity Scale; STDT: Somatosensory temporal discrimination threshold.

Previous studies have shown a significant efficacy of HE program on pain, functionality, and quality of life in patients with FMS.11-13 


\begin{tabular}{|c|c|c|c|c|c|c|c|c|c|}
\hline & Age & $\begin{array}{l}\text { Education } \\
\text { time }\end{array}$ & $\begin{array}{c}\text { The time since } \\
\text { diagnosis }\end{array}$ & VAS & HAD-A & HAD-D & FIQ & SSS & STDT \\
\hline \multicolumn{10}{|l|}{ Age } \\
\hline $\mathrm{r}$ & 1.00 & -0.14 & -0.17 & -0.08 & -0.36 & -0.17 & -0.22 & -0.18 & 0.29 \\
\hline$p$ & & 0.333 & 0.242 & 0.581 & 0.011 & 0.242 & 0.128 & 0.206 & 0.045 \\
\hline \multicolumn{10}{|c|}{ Education time } \\
\hline $\mathrm{r}$ & -0.14 & 1.00 & -0.23 & -0.44 & -0.26 & -0.51 & -0.31 & -0.24 & 0.44 \\
\hline$p$ & 0.333 & & 0.108 & 0.001 & 0.071 & $<0.001$ & 0.031 & 0.088 & 0.767 \\
\hline \multicolumn{10}{|c|}{ The time since diagnosis } \\
\hline$r$ & -0.02 & -0.23 & 1.00 & 0.11 & 0.14 & 0.25 & 0.21 & 0.31 & 0.15 \\
\hline$p$ & 0.847 & 0.108 & & 0.44 & 0.343 & 0.08 & 0.142 & 0.032 & 0.31 \\
\hline \multicolumn{10}{|c|}{ VAS } \\
\hline $\mathrm{r}$ & -0.82 & -0.44 & 0.11 & 1.00 & 0.61 & 0.53 & 0.72 & 0.48 & -0.38 \\
\hline$p$ & 0.581 & 0.001 & 0.44 & & $<0.001$ & $<0.001$ & $<0.001$ & $<0.001$ & 0.799 \\
\hline \multicolumn{10}{|c|}{ HAD-A } \\
\hline r & -0.36 & -0.26 & 0.14 & 0.61 & 1.00 & 0.78 & 0.72 & 0.77 & 0.16 \\
\hline$p$ & 0.011 & 0.071 & 0.343 & $<0.001$ & & $<0.001$ & $<0.001$ & $<0.001$ & 0.258 \\
\hline \multicolumn{10}{|c|}{ HAD-D } \\
\hline $\mathrm{r}$ & -0.17 & -0.51 & 0.25 & 0.53 & 0.78 & 1.00 & 0.65 & 0.64 & 0.08 \\
\hline$p$ & 0.242 & $<0.001$ & 0.08 & $<0.001$ & $<0.001$ & & $<0.001$ & $<0.001$ & 0.568 \\
\hline \multicolumn{10}{|l|}{ FIQ } \\
\hline $\mathrm{r}$ & -0.22 & -0.31 & 0.21 & 0.72 & 0.72 & 0.65 & 1.00 & 0.82 & 0.16 \\
\hline$p$ & 0.128 & 0.031 & 0.142 & $<0.001$ & $<0.001$ & $<0.001$ & & $<0.001$ & 0.253 \\
\hline \multicolumn{10}{|l|}{ SSS } \\
\hline$r$ & -0.18 & -0.24 & 0.31 & 0.48 & 0.77 & 0.64 & 0.81 & 1.00 & 0.26 \\
\hline$p$ & 0.206 & 0.088 & 0.032 & $<0.001$ & $<0.001$ & $<0.001$ & $<0.001$ & & 0.073 \\
\hline \multicolumn{10}{|c|}{ STDT } \\
\hline $\mathrm{r}$ & 0.29 & 0.44 & 0.15 & -0.03 & $\mathrm{r}-0.16$ & 0.08 & 0.16 & 0.26 & 1.00 \\
\hline$p$ & 0.045 & 0.767 & 0.31 & 0.799 & 0.258 & 0.568 & 0.253 & 0.073 & \\
\hline
\end{tabular}

However, compliance with a HE program has been found to be lower than that of SE programs, which has reduced the efficacy of the program. In a study, the reduction in pain was greater in those who completed the HE program, compared to those who did not, highlighting the importance of continuing exercise on pain in FMS patients. ${ }^{14}$ Similarly, in the current study, a significant improvement was found in the frequent symptoms of FMS patients with a HE program. Unlike the above-mentioned study, compliance with the HE program was higher. The rates of withdrawal from the treatment were similar in both groups with three patients leaving the HEG and two patients leaving the SEG. This high rate of compliance was attributed to the short term of the programs applied at only four weeks. Exercises performed under supervision have been reported to be more effective than exercise programs illustrated in a home-based program. ${ }^{15}$ In the current study, both exercise programs were found to have a positive clinical effect on FMS patients; however, these positive clinical effects were significantly greater in SEG. Informing patients in HEG about FMS and explaining the importance of the exercises can lead to an increase in compliance with a $\mathrm{HE}$ program and, thus, to positive effects. However, there is no measurement proving that patients have fully complied with a HEG. Patients may have performed fewer exercises or for a shorter time than instructed and this could cause the lower level of positive clinical effects compared to SEG. It may also be that, in SEG, feedback given by the physiotherapist have encouraged and may have had a positive effect on the group treatment results. 
The chronic pain in FMS patients is thought to be the result of continuing painful stimuli, leading to maladaptive neuroplasticity. ${ }^{16}$ Due to an impairment in central inhibition, there is exaggerated perception of painful stimuli by patients. The importance of the cortical level in central sensitization has been frequently shown in recent studies. Neurological imaging has shown increased activation in the primary somatosensory cortex both during painful stimuli and at rest in FMS. ${ }^{17,18}$ In addition to hyperalgesia in these patients, allodynia is often seen. In other words, there is a problem in the central processing of a painless sensory stimulation. In a previous study, FMS patients were found to be more sensitive to painless mechanical stimulation. Compared to healthy individuals, an increased amplitude was seen in the cortical response of FMS patients with a painless electrical stimulation. ${ }^{19}$

The STD is the ability to perceive two separate stimuli applied at different times. Filtering of the stimuli entering from different sources is essential for normal STD ability. Different STDT values are obtained from different body areas. In several studies conducted on healthy populations, the STDT value has been obtained as approximately $30 \mathrm{~ms}^{20-22}$ In a study which compared FMS patients with a healthy control group, the STDT value was mean $33 \mathrm{msn}$ in the healthy control group and was significantly higher at mean $69 \mathrm{~ms}$ in the FMS patients. ${ }^{6}$ The most likely reason for the high STDT values found in FMS patients is the impairment in the central processing process of painless tactile stimulation such as a pain stimulus. The basal ganglion, cerebellum, and primary somatosensorial cortex are thought to be the areas of the CNS responsible for the STD. ${ }^{4,5,23}$ Areas showing changes in activation in imaging studies of FMS patients are consistent with these areas thought to be responsible for the STD ability. Rocchi et al. ${ }^{5}$ found a relationship between impaired STD ability and increased reaction time in healthy individuals. Prolonged reaction time was reflected as a delay in the decision-making process. Therefore, the impaired STD ability in FMS patients may be related to cognitive dysfunctions, such as impaired ability to make decisions, which is often observed in these patients. ${ }^{24,25}$ Cognitive impairment was previously shown to be associated with central sensitization in FMS. ${ }^{26}$
In our study, the STDT values of FMS patients were found to be high (mean 80 $\mathrm{msn}$ ), which is consistent with the findings of previous studies. Disrupted STD supported an impairment in sensory processing in FMS. Moreover, the increased STDT may be associated with cognitive dysfunction in FMS. However, we were unable to assess cognitive functions with detailed cognitive tests in patients with FMS. At the end of the four-week exercise programs, a statistically significant improvement (mean $7 \mathrm{~ms}$ ) was seen in STDT in both groups. Martinsen et al. ${ }^{27}$ demonstrated that a 15-week exercise program improved cognitive performance in FMS patients. Reduced STDT values after exercise program in our study may indicate an improved cognitive performance in patients with FMS. When the treatment group $\times$ time interaction was examined, no significant difference was found between the groups in respect of STDT improvement. Both the HE and SE programs were effective on STDT, in addition to the clinical parameters, confirming that the efficacy of exercise in the FMS patients was through the CNS. A reduction in the STDT value with exercise may be an indicator of the clinical efficacy of exercise and can be used in clinical studies. However, the higher level of clinical efficacy of the SE program in the current study was not seen on STDT. In addition, no significant correlation was found between the baseline clinical parameters and the baseline STDT values in either group of FMS patients. Therefore, the STDT may not be a very sensitive method to show fibromyalgia symptoms and the effect of exercise on these symptoms.

One of the limitations of the present study is the short duration (four weeks) of the exercise program which could be the reason for the similar positive effect of HEG and SEG on STD in FMS patients. Another limitation is that we were unable to evaluate cognitive function in patients with FMS. Therefore, we cannot conclude whether altered STDT values are the signs of cognitive dysfunction in FMS. The cooperation of the participant is required for STDT measurement. To overcome this limitation, we included patients with at least eight years of formal education.

In conclusion, the results of this study show, for the first time, that a significant improvement 
in impaired STDT can be achieved with SEG. However, this positive effect of SEG on STD does not seem to be superior to HEG. The amelioration in the impaired sensory processing is not sufficient to show the efficacy of exercise on clinical parameters. Further long-term, prospective, randomized-controlled studies are required.

\section{Declaration of conflicting interests}

The authors declared no conflicts of interest with respect to the authorship and/or publication of this article.

\section{Funding}

The authors received no financial support for the research and/or authorship of this article.

\section{REFERENCES}

1. Boomershine CS. Fibromyalgia: the prototypical central sensitivity syndrome. Curr Rheumatol Rev 2015;11:131-45.

2. Sindel D, Saral İ, Esmaeilzadeh S. Fibromiyalji sendromunda uygulanan tedavi yöntemleri. Türk Fiz Tip Rehab Derg 2012;58:136-42.

3. Gowans SE, deHueck A. Effectiveness of exercise in management of fibromyalgia. Curr Opin Rheumatol 2004;16:138-42.

4. Conte A, Modugno N, Lena F, Dispenza S, Gandolfi $\mathrm{B}$, Iezzi $\mathrm{E}$, et al. Subthalamic nucleus stimulation and somatosensory temporal discrimination in Parkinson's disease. Brain 2010;133:2656-63.

5. Rocchi L, Casula E, Tocco P, Berardelli A, Rothwell J. Somatosensory temporal discrimination threshold involves inhibitory mechanisms in the primary somatosensory area. J Neurosci 2016;36:325-35.

6. Gunendi Z, Polat M, Vuralli D, Cengiz B. Somatosensory temporal discrimination is impaired in fibromyalgia. J Clin Neurosci 2019;60:44-8.

7. Aydemir Ö. Guvenir T. Kuey L. Kultur S. Hastane Anksiyete Depresyon Ölçeği Türkçe formunun geçerlilik ve güvenilirlik çalışması. Türk Psikiyatri Dergisi 1997;8:280-7.

8. Sarmer S, Ergin S, Yavuzer G. The validity and reliability of the Turkish version of the Fibromyalgia Impact Questionnaire. Rheumatol Int 2000;20:9-12.

9. Wolfe F, Clauw DJ, Fitzcharles MA, Goldenberg DL, Katz RS, Mease P, et al. The American College of Rheumatology preliminary diagnostic criteria for fibromyalgia and measurement of symptom severity. Arthritis Care Res (Hoboken) 2010;62:600-10.

10. Yanmaz MN, Atar S, Biçer M. The reliability and validity of the Turkish version of fibromyalgia survey diagnostic criteria and symptom severity scale. J Back Musculoskelet Rehabil 2016;29:287-93.
11. Evcik D, Aytaç F. The effects of thearapy in the treatment of primary fibromyalgia patients. Romatizma 2001;16:27-31.

12. Da Costa D, Abrahamowicz M, Lowensteyn I, Bernatsky S, Dritsa M, Fitzcharles MA, et al. A randomized clinical trial of an individualized home-based exercise programme for women with fibromyalgia. Rheumatology (Oxford) 2005;44:1422-7.

13. Luedtke CA, Thompson JM, Postier JA, Neubauer BL, Drach S, Newell L. A description of a brief multidisciplinary treatment program for fibromyalgia. Pain Manag Nurs 2005;6:76-80.

14. Harden RN, Song S, Fasen J, Saltz SL, Nampiaparampil D, Vo A, et al. Home-based aerobic conditioning for management of symptoms of fibromyalgia: a pilot study. Pain Med 2012;13:835-42.

15. Ramsay C, Moreland J, Ho M, Joyce S, Walker $\mathrm{S}$, Pullar T. An observer-blinded comparison of supervised and unsupervised aerobic exercise regimens in fibromyalgia. Rheumatology (Oxford) 2000;39:501-5.

16. Mhalla A, de Andrade DC, Baudic S, Perrot S, Bouhassira D. Alteration of cortical excitability in patients with fibromyalgia. Pain 2010;149:495-500.

17. Cook DB, Lange G, Ciccone DS, Liu WC, Steffener $\mathrm{J}$, Natelson $\mathrm{BH}$. Functional imaging of pain in patients with primary fibromyalgia. J Rheumatol 2004;31:364-78.

18. Kim JY, Kim SH, Seo J, Kim SH, Han SW, Nam EJ, et al. Increased power spectral density in resting-state pain-related brain networks in fibromyalgia. Pain 2013;154:1792-7.

19. Lim M, Roosink M, Kim JS, Kim HW, Lee EB, Son $\mathrm{KM}$, et al. Augmented pain processing in primary and secondary somatosensory cortex in fibromyalgia: a magnetoencephalography study using intra-epidermal electrical stimulation. PLoS One 2016;11:e0151776.

20. Hoshiyama M, Kakigi R, Tamura Y. Temporal discrimination threshold on various parts of the body. Muscle Nerve 2004;29:243-7.

21. Boran HE, Cengiz B, Bolay H. Somatosensory temporal discrimination is prolonged during migraine attacks. Headache 2016;56:104-12.

22. Vuralli D, Boran HE, Cengiz B, Coskun O, Bolay $\mathrm{H}$. Somatosensory temporal discrimination remains intact in tension-type headache whereas it is disrupted in migraine attacks. Cephalalgia 2017;37:1241-7.

23. Morgante F, Tinazzi M, Squintani G, Martino D, Defazio G, Romito L, et al. Abnormal tactile temporal discrimination in psychogenic dystonia. Neurology 2011;77:1191-7.

24. Leavitt F, Katz RS, Mills M, Heard AR. Cognitive and dissociative manifestations in fibromyalgia. $\mathrm{J}$ Clin Rheumatol 2002;8:77-84.

25. Tesio V, Torta DM, Colonna F, Leombruni P, Ghiggia A, Fusaro E, et al. Are fibromyalgia patients cognitively impaired? Objective and subjective 
neuropsychological evidence. Arthritis Care Res (Hoboken) 2015;67:143-50.

26. Coppieters I, Ickmans $\mathrm{K}$, Cagnie $\mathrm{B}$, Nijs J, De Pauw R, Noten Set al. Cognitive Performance Is Related to Central Sensitization and Health-related Quality of Life in Patients with Chronic WhiplashAssociated Disorders and Fibromyalgia. Pain Physician
2015;18:E389-401.

27. Martinsen S, Flodin P, Berrebi J, Löfgren M, Bileviciute-Ljungar I, Mannerkorpi K, et al. The role of long-term physical exercise on performance and brain activation during the Stroop colour word task in fibromyalgia patients. Clin Physiol Funct Imaging 2018;38:508-16. 